\title{
ARTICLES
}

\section{Phase separation in the binary-alloy problem: The one-dimensional spinless Falicov-Kimball model}

\author{
J. K. Freericks \\ Department of Physics, Georgetown University, Washington, DC 20057 \\ Ch. Gruber, and N. Macris \\ Institut de Physique Théorique, Ecole Polytechnique Fédérale de Lausanne, PHB-Ecublens, CH-1015, Lausanne, Switzerland
}

(Received 8 February 1996)

\begin{abstract}
The ground states of the one-dimensional Falicov-Kimball model are investigated in the small-coupling limit, using nearly degenerate perturbation theory. For rational electron and ion densities, respectively, equal to $p / q, p_{i} / q$, with $p$ relatively prime to $q$ and $p_{i} / q$ close enough to $\frac{1}{2}$, we find that in the ground state the ion configuration has a period $q$. The situation is analogous to the Peierls instability, where the usual arguments predict a period- $q$ state that produces a gap at the Fermi level and is insulating. However for $p_{i} / q$ far enough from $\frac{1}{2}$, this phase becomes unstable against phase separation. The ground state is a mixture of a period- $q$ ionic configuration and an empty (or full) configuration, where both configurations have the same electron density to leading order. Combining these results with those previously obtained for strong coupling, it follows that a phase transition occurs in the ground state, as a function of the coupling, for ion densities far enough from $\frac{1}{2}$. [S0163-1829(96)00924-1]
\end{abstract}

\section{INTRODUCTION}

The theory of the electronic band structure of solids is one of the oldest theories in condensed-matter physics, dating back to 1928 when Bloch proved the existence of electronic bands in solids. ${ }^{1}$ It was soon discovered that most metals could be described with the nearly free electron model, where the periodic ion potential felt by the electrons is weak. The initial emphasis in the development of band theory focused on one-dimensional models, where both transfermatrix results ${ }^{2}$ and exact solutions ${ }^{3,4}$ could be found. It was only after the development of the pseudopotential method ${ }^{5}$ in the 1960's that the success of the nearly free electron model was understood.

In the 1950 's, Peierls ${ }^{6}$ reexamined the perturbation theory for the nearly free electron model and found that, in onedimension, a static distortion would always reduce the energy of a solid, because the opening of a gap (at the Fermi level) in the electronic band structure would lower the energy of the occupied electronic states and raise the energy of the unoccupied states. Such a distortion would produce an insulator from the parent metal and Peierls's work led to the conclusion that there can never be a one-dimensional metal. Fröhlich ${ }^{7}$ used nearly degenerate perturbation theory to show that the decrease in the electronic energy was on the order of $\delta^{2} \ln \delta$ for a periodic distortion of amplitude $\delta$, while the elastic energy was on the order of $\delta^{2}$, so the net effect of the distortion was to reduce the ground-state energy (if $\delta$ was small enough). Two chemists, Longuet-Higgins and Salem, ${ }^{8}$ independently arrived at the same conclusions by examining a general class of Hamiltonians for ring-shaped molecules. Note that these conclusions explicitly neglect the quantum fluctuations of the phonons about the distorted state, a feature which can lead to a stabilization of the undistorted phase, as illustrated in recent rigorous work on the onedimensional Holstein model. ${ }^{9}$

The Peierls distortion is generally studied at half filling for the electrons, in which case the distortion leads to a doubling of the unit cell. The conventional wisdom is that the lowest periodic structure that produces a gap at the Fermi level will be the true ground state, or, in other words, the Peierls distortion is stable against any higher-order distortions. This result has been recently proven to be true at half filling. ${ }^{10,11}$

In one dimension, the restriction to half filling is not necessary, with the result that a periodic distortion that produces a gap at the Fermi level will always lower the total energy of the system. Furthermore, one can generalize Peierls' arguments to include other models, such as models for alloy formation. Historically, these alloy models were studied before the models of lattice distortions, where Hume-Rothery ${ }^{12}$ pointed out that certain alloys form only when the electronatom ratio fell within very narrow ranges. In one-dimension, Peierls-like arguments establish the Hume-Rothery rule, since a periodic arrangement of the ions will always produce the largest gap at the Fermi level.

The simplest model of a binary alloy consists of one band of itinerant electrons interacting with static ions:

$$
H=-t \sum_{i=1}^{N}\left(c_{i}^{\dagger} c_{i+1}+c_{i+1}^{\dagger} c_{i}\right)-U \sum_{i=1}^{N} c_{i}^{\dagger} c_{i} w_{i},
$$

where $c_{i}^{\dagger}$ is the creation operator for an electron at site $i$, $w_{i}$ is a classical variable that is 1 if the site is occupied by an 
A ion and 0 if the site is occupied by a B ion. The hopping integral is $t$ and $U$ denotes the difference in on-site energies for an electron on a B site minus that of an electron on an A site. The electron density, $\rho_{e}$, is the number of electrons per site $\left[\rho_{e}=(1 / N) \Sigma_{i}\left\langle c_{i}^{\dagger} c_{i}\right\rangle\right]$, and similarly the ion density $\rho_{i}$ is the number of A ions per site $\left[\rho_{i}=(1 / N) \Sigma_{i} w_{i}\right]$, with $N$ the number of lattice sites. We are interested in the thermodynamic limit, and so we take $N \rightarrow \infty$, but maintain finite values for both $\rho_{e}$ and $\rho_{i}$. In the alloy picture $(U>0)$, one can envision that the electrons are donated by one of the ionic species (say the A ion), in which case a study of the neutral case (where $\rho_{i}=\rho_{e}$ ) becomes most relevant.

This model is a simplified version (spinless, single band) of the model proposed by Falicov and Kimball ${ }^{13}$ to discuss semiconductor-insulator transitions in rare-earth oxides and borides. Later, the same model was employed to study ordering of rare-earth ions in mixed-valence systems. ${ }^{14}$ More recently, ${ }^{15}$ work on the Falicov-Kimball model has focused on another aspect, that of periodic crystal formation. In this language the A sites are ions, and the B sites are empty sites. The question studied is whether the mutual interaction of the ions and electrons, coupled with the fact that the electrons satisfy the Pauli exclusion principle, causes a periodic arrangement of the ions to be the ground-state configuration. This is the language that we adopt in this contribution.

The Hamiltonian in Eq. (1) exhibits two different symmetries: ${ }^{15}$ an ion-occupied-empty-site symmetry and an electron-hole symmetry. The first symmetry relates the ground-state energy (per site) for the configuration of ions $\left\{w_{i}\right\}$ to the energy of the conjugate configuration $\left\{w_{i}^{*}\right\}:=\left\{1-w_{i}\right\}$,

$$
E_{\mathrm{gs}}\left(U, \rho_{e},\left\{w_{i}^{*}\right\}\right)=E_{\mathrm{gs}}\left(-U, \rho_{e},\left\{w_{i}\right\}\right)-U \rho_{e},
$$

while the second symmetry employs the unitary transformation $c_{i} \rightarrow(-1)^{i} d_{i}^{\dagger}$ that changes electrons to holes, yielding

$$
E_{\mathrm{gs}}\left(U, 1-\rho_{e},\left\{w_{i}\right\}\right)=E_{\mathrm{gs}}\left(-U, \rho_{e},\left\{w_{i}\right\}\right)-U \rho_{i} .
$$

These two symmetries allow restriction to the region $\rho_{e} \leqslant \frac{1}{2}$ and $U>0$, without a loss in generality.

In the crystallization picture, the most natural assumption to make is that the number of electrons equals the number of ions, and the system is charge neutral $\left(\rho_{e}=\rho_{i}\right)$. However, the nonneutral case is also of interest, since the electron density can be modified by either doping the system with impurities, or by allowing the system to be coupled to an electron resevoir. The latter picture is important in making contact with quasi-one-dimensional systems, where the neglected bands act as an electron reservoir, allowing charge transfer into or out of the one-dimensional chains.

The Falicov-Kimball model has been actively studied in recent years, ever since Kennedy and Lieb ${ }^{15}$ and Brandt and Schmidt ${ }^{16}$ independently proved that the period-two phase is the ground state for all $U$ when the electron and the ion densities are both equal to $\frac{1}{2}$. Most emphasis has concentrated on the one-dimensional model, where numerical studies ${ }^{17}$ indicated that the system phase separated into the segregated phase (where all the ions cluster on one side of the lattice) for large enough interaction strength if $\rho_{e} \neq \rho_{i}$ or $\rho_{e}+\rho_{i} \neq 1$. In the other cases, where $\rho_{e}=\rho_{i}$ (the neutral case) and $U \rightarrow \infty$, or $\rho_{e}+\rho_{i}=1$ (the mixed-valence case) and
$U \rightarrow-\infty$, it was conjectured that the most homogeneous phase was the ground state. These two conjectures have already been proven to be true. ${ }^{18-20}$ Another conjecture, based upon the many-body version of Rayleigh-Schroedinger perturbation theory, stated that in the small- $U$ limit the groundstate configuration will be the configuration that produces the largest gap at the Fermi level, and this state was shown to have the smallest periodicity that could produce a gap at the Fermi level (consistent with the Peierls picture). Recent analytical $^{21}$ and numerical ${ }^{22}$ work on the neutral case has shown, however, that at low electron density, there is a tendency for molecule formation, rather than a homogeneous distribution of the ions, and a phase-separated configuration of ions may yield a lower energy than a pure periodic phase.

In the spirit of the nearly free electron model, we establish two results in the framework of perturbation theory (which are valid for $U$ sufficiently small): First, we show that if the electron density is $\rho_{e}=(p / q)$ with $p$ relatively prime to $q$, and $\rho_{i}=p_{i} / q_{i}$, with $\left(p^{\prime} / q\right)<\rho_{i}<\left(p^{\prime}+1\right) / q$ for some integer $p^{\prime}$, then the ground-state configuration is a phaseseparated mixture of period- $q$ phases, and possibly the empty (or full) lattice.

The second result is a statement about the stability of the pure period- $q$ phase for $\rho_{e}=p / q$ (with $p$ relatively prime to $q$ ) and $\rho_{i}=p_{i} / q$. For $\rho_{i} \in\left[\rho_{c}, 1-\rho_{c}\right]$ with $\rho_{c} \approx 0.371$ [solution of Eq. (23)], the ground state has period $q$ and is the most homogeneous configuration; it also has the smallest periodicity needed to produce a gap at the Fermi level. On the other hand, if the ion density $\rho_{i}=p_{i} / q$ is smaller than $\frac{1}{4}$ or greater than $\frac{3}{4}$, then the ground state is always a phaseseparated mixture of a phase with $\rho_{e}=p / q, \rho_{i}^{\prime}=0$ (or $\left.\rho_{i}^{\prime}=1\right)$ and a period- $q$ phase with $\rho_{e}=p / q, \rho_{i}^{\prime \prime}=p_{i}^{\prime \prime} / q$ a rational that is closest to $\rho_{c}$ in a well-defined sense. For $\rho_{i}=p_{i} / q<\rho_{c}$ or $\rho_{i}=p_{i} / q>1-\rho_{c}$, the same is true, i.e., the ground state is a phase-separated mixture, except for special values of $\rho_{i}$ [those satisfying Eq. (24)] for which the period$q$ phase is stable.

These results show that the close analogy with the Peierls instability is valid only for $\rho_{c}<\rho_{i}<1-\rho_{c}$. We view the analogy as follows: For $U=0$ (and $\rho_{e}=p / q, \rho_{i}=p_{i} / q$ fixed), any ion configuration is a ground state, i.e., the probability to find an ion at a given site is uniform and equals $\rho_{i}$. This uniform-density state is the "undistorted state," has no gap in the electronic spectrum, and is metallic. For $U$ $\neq 0$, sufficiently small, a particular ion configuration is selected, which has period $q$. It corresponds to the PeierlsFrölich "distorted state," which has a gap at the Fermi level and is insulating. For $\rho_{i}<\rho_{c}$ or $\rho_{i}>1-\rho_{c}$, the ground state is (in general) phase separated and is a mixture of a metallic and an insulating state. This situation does not have a counterpart in the standard theory of Peierls and Fröhlich.

Finally, the above results establish the existence of a phase transition in the ground state of the Falicov-Kimball model when $U$ is varied. For densities such that the ground state is a phase-separated mixture (for $U$ sufficiently small), there must be a phase transition as $U$ increases. Indeed, for $U$ sufficiently large, the ground state is known to be either the most-homogeneous phase or the segregated phase (which is a different phase-separated state). 
Our presentation is organized as follows: in Sec. II the perturbation theory is developed showing the $U^{2} \ln U$ behavior of the ground-state energy for small $U$; in Sec. III the perturbation-theory results are analyzed to show when pure phases are the ground state and when the ground state is phase separated; a discussion follows in Sec. IV.

\section{PERTURBATION THEORY}

It is most convenient to rewrite the Falicov-Kimball Hamiltonian in a momentum-space representation before developing a perturbation-series expansion for the ground-state energy. Using the standard Fourier transform

$$
a_{k}:=\frac{1}{\sqrt{N}} \sum_{j=1}^{N} e^{-i k j} c_{j}
$$

(with the lattice spacing set equal to 1) yields

$$
H=\sum_{k}[\epsilon(k)-U W(0)] a_{k}^{\dagger} a_{k}-U \sum_{k \neq k^{\prime}} W\left(k-k^{\prime}\right) a_{k}^{\dagger} a_{k^{\prime}}
$$

for the Hamiltonian of the Falicov-Kimball model in momentum space. The wave vectors $k$ and $k^{\prime}$ are restricted to the first Brillouin zone $(-\pi<k \leqslant \pi)$ and $\epsilon(k):=-2 t \cos k$ is the unperturbed band structure. $W(2 \pi n / Q)$ is the structure factor of the period- $Q$ ion configuration $\left\{w_{i}\right\}$,

$$
W(2 \pi n / Q):=\frac{1}{Q} \sum_{j=1}^{Q} e^{-i(2 \pi n j / Q)} w_{j},
$$

defined for $n=0,1, \ldots, Q-1$. (It is notationally simpler here to define the $k$ vectors with $k=2 \pi n / Q$ to sometimes lie outside of the first Brillouin zone. Of course, translation by $-2 \pi$ will shift these vectors back into the first Brillouin zone.) Note that $W(0)=\rho_{i}$ by definition.

We begin by performing the many-body version of Rayleigh-Schrödinger perturbation theory with the doublesummation term in Eq. (5) acting as the perturbation. The analysis is straightforward, ${ }^{17}$ requiring a momentum-space integral that can be evaluated analytically, yielding

$$
\begin{aligned}
E_{\mathrm{gs}}\left(U, \rho_{e},\left\{w_{i}\right\}\right)= & -\frac{2 t}{\pi} \sin \left(\pi \rho_{e}\right)-U \rho_{e} \rho_{i} \\
& +\frac{U^{2}}{8 \pi t} \sum_{n=1} \frac{|W(2 \pi n / Q)|^{2}}{\sin (\pi n / Q)} \\
& \times \ln \left|\frac{\sin (\pi n / Q)-\sin \left(\pi \rho_{e}\right)}{\sin (\pi n / Q)+\sin \left(\pi \rho_{e}\right)}\right|+O\left(U^{3}\right)
\end{aligned}
$$

for the ground-state energy of configuration $\left\{w_{i}\right\}$.

The perturbative expansion in Eq. (7) has a singularity when the electron density is rational $\rho_{e}=p / q$ and the ion configuration has a period that is a multiple of $q$ (with the exception of those ion configurations, for which the relevant structure factor vanishes). It was argued heuristically in Ref. 17 that the configuration with the maximal singularity (i.e., with the maximal value of $\left|W\left(2 \pi \rho_{e}\right)\right|$ ) will be the groundstate configuration, and this result agreed with the numerical work. However, such logic is flawed, because the expansion in Eq. (7) is valid for $U / t \ll|\ln | \sin (\pi n / Q)-\sin \left(\pi \rho_{e}\right)||$, which cannot hold when an integral number of electronic subbands are filled [i.e., when $\left.\rho_{e}=(p / q)\right]$. This result was known by Fröhlich, ${ }^{7}$ and it arises from the fact that there are degeneracies in the unperturbed wave function that were neglected in the above analysis.

It is easiest to see the origin of the degeneracies and how to properly treat them by examining the perturbation theory of the single-particle energy levels. Wigner-Brillouin perturbation theory is used, because it automatically removes the singularities. The ground-state energy is found by simply filling up the lowest available single-particle energy levels in the system. These energy levels can be expanded in a perturbation series, which yields

$$
E\left(k, U,\left\{w_{i}\right\}\right)=\epsilon(k)+\frac{U^{2}{ }^{Q-1}}{t} \sum_{n=1} \frac{|W(2 \pi n / Q)|^{2}}{E\left(k, U,\left\{w_{i}\right\}\right)-\epsilon\left(k+\frac{2 \pi n}{Q}\right)},
$$

to second order in $U$. The quasiparticle energy $E\left(k, U,\left\{w_{i}\right\}\right)$ appears on both sides of Eq. (8), because one must self-consistently solve for the energy in a WignerBrillouin perturbation-theory expansion. The equivalent Rayleigh-Schrödinger expansion would replace $E(k)$ by $\epsilon(k)$ in the right hand side of (8), which produces a singularity when $k=-\pi n / Q$, because $\epsilon(k)=\epsilon(-k)$.

At this point, textbooks note that the dominant term in the sum over $n$, in the right-hand side of Eq. (8), is the term where $k+2 \pi n / Q$ is closest to $2 \pi-k$, i.e., it is the term with $n$ closest to $Q(1-k / \pi)$. If the other terms are neglected, then Eq. (8) reduces to a quadratic equation that can be solved exactly. This procedure is sometimes called nearly degenerate perturbation theory, because it produces the correct secular equation in the degenerate case.

However, we choose to proceed in a more precise manner in the case where the value of the interaction is much smaller than the subband width $U \ll \pi t / Q$. In this case, the effect of the additional terms can be treated in a perturbative fashion, which gives

$$
\begin{aligned}
E\left(k, U,\left\{w_{i}\right\}\right)= & -t\left[\cos k+\cos \left(k-\frac{2 \pi n}{Q}\right)\right]+\frac{U^{2}}{t} f_{n}(k) \\
& \pm \sqrt{\left\{t\left[\cos k-\cos \left(k-\frac{2 \pi n}{Q}\right)\right]-\frac{U^{2}}{t} f_{n}(k)\right\}^{2}+\frac{U^{2}}{t}\left|W\left(\frac{2 \pi n}{Q}\right)\right|^{2}}+O\left(U^{3}\right),
\end{aligned}
$$


with

$$
f_{n}(k):=-\frac{1}{4} \sum_{\substack{m=1 \\ m \neq n}}^{Q-1} \frac{\left|W\left(\frac{2 \pi m}{Q}\right)\right|^{2}}{\cos k-\cos \left(k-\frac{2 \pi m}{Q}\right)}
$$

for $\pi\left(n-\frac{1}{2}\right) / Q<k<\pi\left(n+\frac{1}{2}\right) / Q$. The minus sign is for the subband energy with $k \rightarrow \pi n / Q$ from below, and the plus sign is for $k \rightarrow \pi n / Q$ from above. This form for the quasiparticle energies is exact for all $U$ when $Q=2$, but is perturbative for all higher periods.

The ground-state energy is found by summing up all of the quasiparticle energies with $|k|<k_{F}=\pi \rho_{e} \quad\left(k_{F}\right.$ is the Fermi wave vector). Since the quasiparticle energies reproduce the noninteracting result when $U=0$, the zeroth- and first-order terms are correctly produced by this summation. We want to concentrate on the higher-order terms. The solution for the quasiparticle energies reveals that a generic period- $Q$ configuration will break into $Q$ subbands. The band gaps are equal to $2 U|W(2 \pi n / Q)|$ and are symmetrically displaced to lowest order; the order $U^{2}$ correction leads to asymmetries in the subband structure. If the Fermi energy lies within a subband, then it is easy to show that for $U \ll \pi t / Q$ the shift in the ground-state energy is of order
$U^{2} / t$, because the square root in Eq. (9) can always be expanded in a convergent power series in $U$. However, no such perturbation-series expansion can be made if the Fermi energy lies within one of the band gaps. In this case, the ground-state energy actually has a $U^{2} \ln U$ dependence, ${ }^{7}$ which is always larger than any order $U^{2}$ dependence for small enough $U$.

We illustrate the origin of the $U^{2} \ln U$ terms in the expansion for the ground-state energy for rational electron densities $\rho_{e}=(p / q)$ with $p$ relatively prime to $q$. We consider any ion configuration with a period $Q$ that is a multiple of $q$. This guarantees that there will be a band gap at the Fermi momentum $k_{F}=\pi \rho_{e}$. The ground-state energy is

$$
\begin{aligned}
E_{\mathrm{gs}}\left(U, \rho_{e},\left\{w_{i}\right\}\right) & =\sum_{|k|<k_{F}} E\left(k, U,\left\{w_{i}\right\}\right) \\
& =\frac{1}{\pi} \int_{0}^{\pi \rho_{e}} E\left(k, U,\left\{w_{i}\right\}\right) d k .
\end{aligned}
$$

Since the band gaps are symmetric to lowest order, the effects of the lower filled subbands cancel, and the $U^{2} \ln U$ contribution arises entirely from filling the uppermost subband. Therefore, the $U^{2} \ln U$ contribution comes from the integral

$$
I:=-\frac{1}{\pi} \int_{\pi\left[\rho_{e}-(1 / 2 Q)\right]}^{\pi \rho_{e}} \sqrt{\left\{t\left[\cos k-\cos \left(k-2 \pi \rho_{e}\right)\right]-\frac{U^{2}}{t} f_{p}(k)\right\}^{2}+\frac{U^{2}}{t}\left|W\left(2 \pi \rho_{e}\right)\right|^{2}} d k .
$$

Use of the identity $\cos k-\cos \left(k-2 \pi \rho_{e}\right)=-2 \sin \pi \rho_{e} \sin \left(k-\pi \rho_{e}\right)$ and shifting the integration range $k \rightarrow-k+\pi \rho_{e}$ yields

$$
I=-\frac{1}{\pi} \int_{0}^{\pi / 2 Q} \sqrt{\left[2 t \sin \pi \rho_{e} \sin k-\frac{U^{2}}{t} f_{p}\left(\pi \rho_{e}-k\right)\right]^{2}+U^{2}\left|W\left(2 \pi \rho_{e}\right)\right|^{2}} d k .
$$

The $U^{2} \ln U$ behavior originates from the region near the origin and $f_{p}\left(\pi \rho_{e}-k\right)$ does not depend strongly upon $k$ in this region, so we can approximate the integral by replacing $\sin k \rightarrow k$ and $f_{p}\left(\pi \rho_{e}-k\right) \rightarrow f_{p}\left(\pi \rho_{e}\right)$. The substitution $k \rightarrow\left[U\left|W\left(2 \pi \rho_{e}\right)\right| \sinh x+\left(U^{2} / t\right) f_{p}\right] /\left(2 t \sin \pi \rho_{e}\right)$ yields an integrable form for $I$, which contains a constant term and a $U^{2} \ln U$ term. The small- $U$ expansion for the ground-state energy then becomes

$$
\begin{aligned}
E_{\mathrm{gs}}\left(U, \rho_{e},\left\{w_{i}\right\}\right)= & -\frac{2 t}{\pi} \sin \pi \rho_{e}-U \rho_{e} \rho_{i} \\
& +\frac{1}{4 \pi t} \frac{\left|W\left(2 \pi \rho_{e}\right)\right|^{2}}{\sin \pi \rho_{e}} U^{2} \ln U+O\left(U^{2}\right),
\end{aligned}
$$

which contains no $f_{p}$ dependence. The above form is only valid for $U \ll \pi t / Q$. This perturbative expansion shows that the ground state will be found by determining the periodic configuration $\left\{w_{i}\right\}$ that maximizes the square of the structure factor $\left|W\left(2 \pi \rho_{e}\right)\right|^{2}$ at twice the Fermi momentum. Furthermore, it eliminates all configurations with periods $Q$ that are not multiples of $q$, since those states only have a $U^{2}$ correction to their ground-state energy, because the Fermi level does not lie within a subband gap.

\section{PHASE-SEPARATION ANALYSIS}

We are interested in finding the ground state of the Falicov-Kimball model as a function of the electron and ion densities. The analysis is based on the expression (14) of the ground-state energy and is exact in the framework of perturbation theory. To make the results of this section rigorous, we would have to prove that the remainder term in Eq. (14) is indeed $O\left(U^{2}\right)$ uniformly in $q$ and $\left\{w_{i}\right\}$.

The perturbative expansion in Eq. (14) depends on $\rho_{e}$ in the zeroth-order term, which is a convex function of the electron density. Therefore, for $U=0$, phase separation can only occur between two different ion configurations that have the same electron density $\rho_{e}$ as the pure phase.

Let us examine the effect of the first-order term. To order $U$, Eq. (14) is a concave function of $\left(\rho_{e}, \rho_{i}\right)$ and thus the ground state will be a mixture of two phases with densities $\left(\rho_{e}^{\prime}, \rho_{i}^{\prime}\right)$ and $\left(\rho_{e}^{\prime \prime}, \rho_{i}^{\prime \prime}\right)$. We set $\rho_{e}=\alpha \rho_{e}^{\prime}+(1-\alpha) \rho_{e}^{\prime \prime}$, 
$\rho_{i}=\alpha \rho_{i}^{\prime}+(1-\alpha) \rho_{i}^{\prime \prime}$, and $\rho_{e}^{\prime}=\rho_{e}+\delta \rho_{e}$. Since $-\sin \left(\pi \rho_{e}\right)$ is convex, then for $U=0$, we have $\delta \rho_{e}=0$ and the probability that a given site is occupied by an ion is $\rho_{i}$. Hence, $\delta \rho_{e}$ tends to zero as $U \rightarrow 0$. Furthermore, one can check that the minimum of

$$
\alpha E\left(\rho_{e}^{\prime}, \rho_{i}^{\prime}\right)+(1-\alpha) E\left(\rho_{e}^{\prime \prime}, \rho_{i}^{\prime \prime}\right)
$$

(at first order) is attained for

$$
\delta \rho_{e}=\frac{U \rho_{i}}{2 t \sin \left(\pi \rho_{e}\right)}, \quad \rho_{i}^{\prime \prime}=0,
$$

and the decrease in the ground-state energy is of the order $U^{2}$. This is negligible in comparison to the $U^{2} \ln U$ term, so one can assume $\delta \rho_{e}=0$ at this order.

It is the coefficient of the $U^{2} \ln U$ term that determines which ion configuration yields the lowest energy. Since the electron density is fixed in all candidate ground-state configurations, the criterion for selecting the ground-state configuration is to maximize the square of the structure factor $\left|W\left(2 \pi \rho_{e},\left\{w_{i}\right\}\right)\right|^{2}$, including the possibility that phaseseparated mixtures may be needed in the maximization.

The construction of the maximum square structure factor is a straightforward exercise for each phase $\left\{w_{i}\right\}$. Consider a rational electron density $\rho_{e}=(p / q)$ with $p$ relatively prime to $q$ and a rational ion density $\rho_{i}=\left(p_{i} / q_{i}\right)$ with $p_{i}$ relatively prime to $q_{i}$. Then the maximum of $|W|^{2}$ is achieved with the following period- $Q$ ion configuration $^{17}$ (with $\left.Q=\operatorname{lcm}\left\{q, q_{i}\right\}=: s q\right)$. We define the $q$ numbers $r_{j}$ by

$$
\left(p r_{j}\right):=j \bmod q, j=0,1, \ldots, q-1
$$

and set $w_{i}=1$ for

$$
\begin{aligned}
\quad i=r_{j}+m q, \quad j=0,1, \ldots, n-1, \quad m=0,1, \ldots, s-1, \quad n=\operatorname{int}\left[q \rho_{i}\right], \\
i=r_{n}+m q, \quad m=\operatorname{any}\left(Q \rho_{i}-s n\right) \text { numbers in the set }\{0,1, \ldots, s-1\} .
\end{aligned}
$$

Note that the above construction is not necessarily unique when $s \neq 1$, but every configuration constructed in such a fashion will have the same square structure factor (the order $U^{2}$ corrections to the energy should split any remaining degeneracies). It is easy to verify that the above construction does satisfy $W(0)=\rho_{i}$ and

$$
\begin{aligned}
\left|W\left(2 \pi \rho_{e}, \rho_{i}\right)\right|^{2} & =\frac{1}{Q^{2}} \sum_{j, k=1}^{Q} w_{j} w_{k} \cos \left[2 \pi \rho_{e} \frac{(j-k)}{Q}\right] \\
& =\frac{n-q \rho_{i}+\left(n-q \rho_{i}\right)^{2}}{q^{2}}+\frac{1}{2 q^{2}} \frac{1+\left(q \rho_{i}-n-1\right) \cos (2 \pi n / q)-\left(q \rho_{i}-n\right) \cos [2 \pi(n+1) / q]}{\sin ^{2} \pi / q} .
\end{aligned}
$$

In the special case where $s=1$, so that $n=q \rho_{i}$, the above form simplifies to

$$
\begin{aligned}
\left|W\left(2 \pi \rho_{e}, \rho_{i}\right)\right|^{2} & =\frac{1}{2 q^{2}} \frac{\left(1-\cos 2 \pi \rho_{i}\right)}{\sin ^{2} \pi / q} \\
& =\frac{1}{q^{2}} \frac{\sin ^{2} \pi \rho_{i}}{\sin ^{2} \pi / q} \quad(s=1) .
\end{aligned}
$$

Note that $\left|W\left(2 \pi \rho_{e}, \rho_{i}\right)\right|^{2}$ depends on $\rho_{e}$ only through the denominator $q$. This fact greatly simplifies the analysis below.

The ion configuration that maximizes the square of the structure factor is identical to Lemberger's mosthomogeneous configuration ${ }^{20}$ in the neutral case $\rho_{i}=\rho_{e}$. In the nonneutral cases, the maximal ion configuration satisfies uniform-distribution properties ${ }^{17}$ in which the configuration is composed of clusters of ions, with only islands of size $l$ and $l-1$ appearing. Furthermore, these islands are "mosthomogeneously" distributed (the most-homogeneous configuration is the special case with islands of size 1).

Lemma (local convexity of the squared structure factor). Assume that the electron density is rational $\rho_{e}=(p / q)$ with $p$ relatively prime to $q$, and that the ion density is also rational $\rho_{i}=\left(p_{i} / q_{i}\right)$ with $p_{i}$ relatively prime to $q_{i}$, and sat- isfies $p^{\prime} / q<\rho_{i}<\left(p^{\prime}+1\right) / q$ for some integer $p^{\prime}$. Then a mixture of ionic phases with ion densities $p^{\prime} / q$ and $\left(p^{\prime}+1\right) / q$ will have a larger square structure factor than the pure phase with ion density $\rho_{i}$.

Proof: We need to show that the maximal square structure factor in Eq. (19) is locally convex. To do this we must examine the condition for convexity, by computing

$$
\begin{aligned}
C= & \left(p^{\prime}+1-q \rho_{i}\right)\left|W\left(2 \pi \rho_{e}, \frac{p^{\prime}}{q}\right)\right|^{2} \\
& +\left(q \rho_{i}-p^{\prime}\right)\left|W\left(2 \pi \rho_{e}, \frac{p^{\prime}+1}{q}\right)\right|^{2} \\
& -\left|W\left(2 \pi \rho_{e}, \rho_{i}\right)\right|^{2} .
\end{aligned}
$$

If $C>0$, then the square structure factor is locally convex, and the lemma will have been proven. Substituting Eqs. (19) and (20) into Eq. (21) yields $C=\left[q \rho_{i}-p^{\prime}\right.$ $\left.+\left(q \rho_{i}-p^{\prime}\right)^{2}\right] / q^{2}$, which is greater than zero for $0<q \rho_{i}-p^{\prime}<1$, which is a condition that holds by hypothesis. Q.E.D.

Comment. The above lemma shows that the search for a maximal square structure factor can be limited to those ion configurations that possess the minimal periodicity $q$ needed to produce a gap at the Fermi level. It has not determined the 
global maximum. That search will be completed below. The lemma does allow us to immediately obtain in the framework of perturbation theory our first result about phase separation in the Falicov-Kimball model.

Property 1 (minimal-period phase separation). If the electron and ion densities satisfy the hypothesis of the lemma, then for $U$ sufficiently small (i.e., $U q \ll 1$ ), the ground-state configuration is a mixture of two period-q phases, with densities $\rho_{i}^{\prime}=\left(p^{\prime} / q\right)$ and $\rho_{i}^{\prime \prime}=\left(p^{\prime \prime} / q\right)\left(p^{\prime}\right.$ or $p^{\prime \prime}$ can be equal to 0 or $q$ ).

Indeed, the perturbative analysis of Sec. II established that in the limit $U \rightarrow 0$, the ground-state configuration is determined by maximizing the square of the structure factor evaluated at twice the Fermi wave vector. The above lemma shows that such a search can be limited to a search over ion configurations with $\rho_{i}^{\prime}=\left(p^{\prime} / q\right), p^{\prime}=0, \ldots, q$. This means that if the ion density does not equal $p^{\prime} / q$, then it must phase separate into a mixture of states, that have electron density of $\rho_{e}=(p / q)$ and ion densities $p^{\prime} / q$ and $p^{\prime \prime} / q$. It follows from the lemma that we only need to search for the ground state among the ion configurations with period $q$ [given $\rho_{e}=(p / q)$ with $p$ relatively prime to $\left.q\right]$, therefore $s=1$ and the square structure factor is given by Eq. (20).

The function $\left(\cos 2 \pi \rho_{i}-1\right)$ is concave for $\rho_{i} \in\left[0, \frac{1}{4}\right] \cup$ $\left[\frac{3}{4}, 1\right]$ and convex for $\rho_{i} \in\left[\frac{1}{4}, \frac{3}{4}\right]$. Hence, if $\rho_{i}$ lies in the interval $\left[0, \frac{1}{4}\right] \cup\left[\frac{3}{4}, 1\right]$, the pure-phase cannot be stable against phase separation.

The convex envelope of the function $\left(\cos 2 \pi \rho_{i}-1\right)$ is given by

$$
\begin{gathered}
\left(\cos 2 \pi \rho_{c}-1\right) \frac{\rho_{i}}{\rho_{c}} \text { for } 0 \leqslant \rho_{i} \leqslant \rho_{c}, \\
\left(\cos 2 \pi \rho_{i}-1\right) \text { for } \rho_{c} \leqslant \rho_{i} \leqslant 1-\rho_{c}, \\
\left(\cos 2 \pi \rho_{c}-1\right) \frac{1-\rho_{i}}{\rho_{c}} \text { for } 1-\rho_{c} \leqslant \rho_{i} \leqslant 1,
\end{gathered}
$$

where $\rho_{c} \approx 0.3710$ is the solution to the equation

$$
2 \pi \rho_{c}=\tan \pi \rho_{c} .
$$

Thus, if $\rho_{i}$ is a rational in the interval $\left[\rho_{c}, 1-\rho_{c}\right]$, the pure phase with $\rho_{e}=(p / q), \rho_{i}=\left(p_{i} / q\right)$ is stable.

Let us now analyze what happens for densities $\rho_{i}$ in the interval $\left[0, \rho_{c}\right]$. The case $\left[1-\rho_{c}, 1\right]$ is similar. The lemma states that we must consider only the ion densities in the discrete set $\left\{\rho_{i}=\left(p^{\prime} / q\right)\right\}$. Given $\rho_{e}=(p / q)$, let $\left(\widetilde{p_{i}} / q\right)$ be the largest rational in the set $\left\{p^{\prime} / q\right\}$ which is smaller than $\rho_{c}$. From the construction of the convex envelope, for any $\rho_{i}<\widetilde{p_{i}} / q$ we know that the ground-state configuration is a mixture of the empty configuration $\rho_{i}^{\prime}=0$ and a period- $q$ configuration with density $\rho_{i}^{\prime \prime}=\left(\widetilde{p_{i}} / q\right)$ or $\left(\widetilde{p_{i}}+1\right) / q$.

To decide between the two possible values of $\rho_{i}^{\prime \prime}$, we have to determine whether $\widetilde{p_{i}} / q$ corresponds to a pure phase, or a mixture of the empty state and a period- $q$ configuration with density $\left(\widetilde{p_{i}}+1\right) / q$. Using Eqs. (14) and (20), it follows that the pure phase $\widetilde{p_{i}} / q$ is stable if

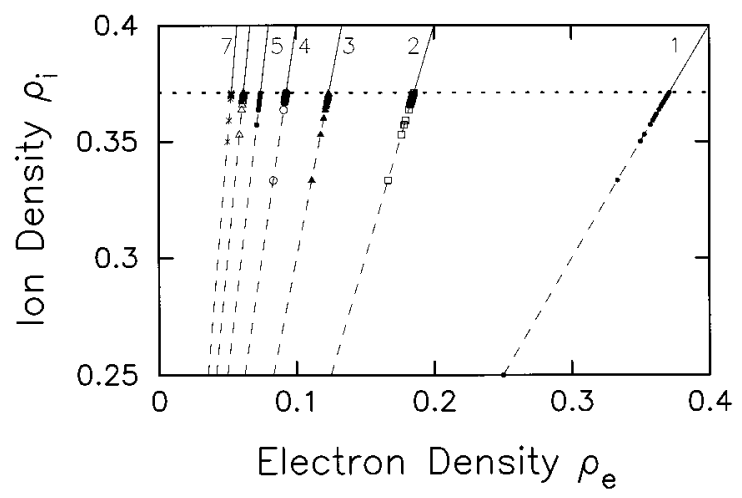

FIG. 1. Stable periodic configuration for $n$ molecules, i.e., states with $\rho_{i}=n \rho_{e}$. The values $n=1$ (solid dot), $n=2$ (open square), $n=3$ (solid triangle), $n=4$ (open dot), $n=5$ (solid square), $n=6$ (open triangle), and $n=7(\mathrm{x})$ are all plotted. The phases are stable above $\rho_{c}$ as indicated by the solid lines. The dashed lines are guides to the eye.

$$
\sin \pi \frac{\widetilde{p_{i}}}{q}>\left(\frac{\widetilde{p_{i}}}{\widetilde{p_{i}}+1}\right)^{1 / 2} \sin \pi \frac{\widetilde{p_{i}}+1}{q},
$$

and unstable if Eq. (24) is not satisfied.

To summarize, given $p / q$ and $\widetilde{p_{i}} / q$, the largest rational with denominator $q$ that is smaller than $\rho_{c}$, if Eq. (24) is satisfied, then for $\rho_{i}=\widetilde{p_{i}} / q$ [respectively, $1-\left(\widetilde{p_{i}} / q\right)$ ] the ground-state configuration is periodic, given by Eq. (17), and for all $\rho_{i}<\left(\widetilde{p_{i}} / q\right)$ the ground state is a mixture with $\rho_{i}^{\prime}=0$ and $\rho_{i}^{\prime \prime}=\left(\widetilde{p_{i}} / q\right)$ [respectively, for all $\rho_{i}>1-\left(\widetilde{p_{i}}+1\right) / q$, $\rho_{i}^{\prime}=1$ and $\left.\rho_{i}^{\prime \prime}=1-\left(\widetilde{p_{i}} / q\right)\right]$. On the other hand, if Eq. (24) is not satisfied, then for all $\rho_{i}<\left(\widetilde{p_{i}}+1\right) / q$ the ground state is a mixture with $\rho_{i}^{\prime}=0$ and $\rho_{i}^{\prime \prime}=\left(\widetilde{p_{i}}+1\right) / q$, and similarly for $\rho_{i}>1-\left(\widetilde{p_{i}} / q\right)$.

In Table I, we give the values of $\widetilde{p_{i}}$ for $q=3-34$, and indicate whether the pure phase with $\rho_{i}=\widetilde{p_{i}} / q$ is stable $(s)$ or unstable $(u)$. For example, the state with $\rho_{e}=\frac{4}{15}$ is unstable for any $\rho_{i}<\frac{6}{15}$ or $\rho_{i}>\frac{9}{15}$ and stable for $\rho_{i}=\frac{6}{15}, \frac{7}{15}, \frac{8}{15}$, $\frac{9}{15}$. The state with $\rho_{e}=\frac{2}{9}$ is unstable for $\rho_{i}<\frac{3}{9}$ or $\rho_{i}>\frac{6}{9}$ and stable for $\rho_{i}=\frac{3}{9}, \frac{4}{9}, \frac{5}{9}, \frac{6}{9}$. In these two examples, the neutral state $\rho_{e}=\rho_{i}$ is unstable. On the other hand, for $\rho_{e}=\frac{4}{11}<\rho_{c}$, the neutral state is stable.

In general, the neutral state $\rho_{e}=\rho_{i}$ is unstable for $\rho_{i}<\rho_{c}$, with an infinite number of exceptions [given by Eq. (24)] for which the first few electron densities are $\rho_{e}=\frac{1}{3}, \frac{1}{4}$, $\frac{4}{11}, \frac{5}{14}, \frac{6}{17}, \frac{7}{19}, \frac{7}{20}, \frac{9}{25}, \frac{10}{27}, \frac{11}{30}$. The state with diatomic molecules $\rho_{i}=2 \rho_{e}$ is unstable for $\rho_{i}<\rho_{c}$ with an infinite number of exceptions $\rho_{e}=\frac{1}{6}, \frac{2}{11}, \frac{3}{17}, \frac{5}{27}, \frac{5}{28}, \frac{7}{38}, \ldots$ Similarly, in the triatomic case $\rho_{i}=3 \rho_{e}$, the exceptional electronic densities for which the pure state is stable are $\rho_{e}=\frac{1}{9}, \frac{2}{17}, \frac{3}{25}, \frac{4}{33}, \ldots$ In any case, it appears that for any $\epsilon>0$ and for any state with $n$ molecules $\rho_{i}=n \rho_{e}$, there is a finite number of exceptions in $\left[\frac{1}{4}, \rho_{c}-\epsilon\right]$ as shown in Fig. 1 .

These observations lead us to the following result:

Property 2. If the electron density is rational, $\rho_{e}=(p / q)$, with $p$ relatively prime to $q$, and the ion density is $\rho_{i}=\left(p_{i} / q\right)$. Then, 
TABLE I. Largest integer $\widetilde{p_{i}}$, such that $\left(\widetilde{p_{i}} / q\right)<\rho_{c} \approx 0.371$. The letters $s$ and $u$ denote whether Eq. ( 24) is satisfied ( $s$ ), implying the pure phase $\rho_{i}=\left(\widetilde{p_{i}} / q\right)$ is stable, or is not satisfied $(u)$, implying the pure phase $\rho_{i}=\left(\widetilde{p_{i}} / q\right)$ is unstable.

\begin{tabular}{cccccccccccccccccccccccccccccccccccccc}
\hline \hline$q$ & 3 & 4 & 5 & 6 & 7 & 8 & 9 & 10 & 11 & 12 & 13 & 14 & 15 & 16 & 17 & 18 & 19 & 20 & 21 & 22 & 23 & 24 & 25 & 26 & 27 & 28 & 29 & 30 & 31 & 32 & 33 & 34 \\
\hline$\widetilde{p_{i}}$ & 1 & 1 & 1 & 2 & 2 & 2 & 3 & 3 & 4 & 4 & 4 & 5 & 5 & 5 & 6 & 6 & 7 & 7 & 7 & 8 & 8 & 8 & 9 & 9 & 10 & 10 & 10 & 11 & 11 & 11 & 12 & 12 \\
& $s$ & $s$ & $u$ & $s$ & $u$ & $u$ & $s$ & $u$ & $s$ & $s$ & $u$ & $s$ & $u$ & $u$ & $s$ & $u$ & $s$ & $s$ & $u$ & $s$ & $u$ & $u$ & $s$ & $u$ & $s$ & $s$ & $u$ & $s$ & $u$ & $u$ & $s$ & $u$ \\
\hline \hline
\end{tabular}

(a) For $\left(p_{i} / q\right) \in\left[\rho_{c}, 1-\rho_{c}\right]$, or $\left(p_{i} / q\right)=\left(\widetilde{p_{i}} / q\right)$ with $\tilde{p_{i}}$ solving Eq. (24), the ground-state configuration is periodic with period $q$.

(b) For $\left(p_{i} / q\right)<\rho_{c} \quad\left(\right.$ or $\left.\left(p_{i} / q\right)>1-\rho_{c}\right)$ and $\left(p_{i} / q\right)$ $\neq\left(\widetilde{p_{i}} / q\right)$ with $\widetilde{p_{i}}$ solving Eq. (24), the ground-state configuration is a mixture of the empty lattice $\rho_{i}^{\prime}=0$ and the period$q$ configuration with $\rho_{i}^{\prime \prime}=\left(\widetilde{p_{i}}+1\right) / q$, (respectively, $\rho_{i}^{\prime}=1$ and $\left.\rho_{i}^{\prime \prime}=1-\left[\left(\widetilde{p_{i}}+1\right) / q\right]\right)$.

(c) For all $\left(p_{i} / q\right)<\frac{1}{4}$ or $\left(p_{i} / q\right)>\frac{3}{4}$, the ground state configuration is a mixture like in $(b)$.

Comments: (i) The exceptional ion densities can all be found by studying Eq. (24). We have not been able to determine an explicit formula for these exceptional ion densities. (ii) The phase-separated state is not an insulating state, but rather is the mixture of a metallic state (the empty lattice) and an insulating state (the period- $q$ phase with $p$ filled subbands). (iii) In the neutral case, $\rho_{e}=\rho_{i}=\rho$, for any $\rho$ $\in\left[\rho_{c}, 1-\rho_{c}\right]$ and for the "exceptional", values in the intervals $\left[0, \rho_{c}\right]$ or $\left[1-\rho_{c}, 1\right]$, the ground state is most homogeneous, since the state with the maximal structure factor satisfies the uniform-distribution property. It is also the configuration obtained by Lemberger's construction. ${ }^{20}$ For these pure states, it is expected that the ground state does not have any phase transition when $U$ increases from +0 to $+\infty$, since, for any rational density $\rho$, the ground state is known to be the most homogeneous state for $U$ sufficiently large. This expectation is also confirmed for intermediate values of $U(U \geqslant 0.1)$ by exact numerical calculations. ${ }^{22}$ Using the same argument for the "regular' values of $\rho$ in $\left[0, \rho_{c}\right]$ or $\left[1-\rho_{c}, \rho_{c}\right]$, there will be a phase transition as $U$ varies. (iv) These results only hold for $U$ sufficiently small with respect to $1 / q$, where $\rho_{e}=(p / q)$, for the $U^{2} \ln U$ term to dominate the perturbation expansion. For $\rho_{e}=(p / q)$ and $\rho_{i}=\left(p_{i} / q\right) \neq \rho_{e}$, the phase separation that may occur for small $U$ rapidly disappears as $U$ is increased from 0 to $\infty$ to yield either a pure state or the segregated phase. ${ }^{22}$ For $U$ sufficiently large, it is expected that the state is either neutral or the segregated phase.

\section{CONCLUSION}

The band theory of solids is perhaps the defining theory for condensed-matter physics. It has been applied to virtually every interesting material that has been studied. Nevertheless, the conventional wisdom of Peierls and Fröhlich for optimizing the band structure for the ground state of onedimensional crystals is not always correct. They argue, that the ion configuration that produces the largest gap at the Fermi level will yield the ground state. We find that this argument is true for a nearly free electron model only if the ion density is close enough to half filling. For ion densities away from half filling, the system will phase separate into a mixture of states that have the same electron density, but have different ion densities $\left(\rho_{i}=0\right.$ and $\rho_{i}$ close to 0.371 or $\rho_{i}=1$ and $\rho_{i}$ close to 0.629$)$. It is possible that this phase separation can be observed in quasi-one-dimensional metals and insulators. We are not aware of any experiments that have seen this phase separation. In fact, because entropy effects will suppress such phase separation at finite temperatures, it may be problematic to observe this behavior experimentally.

Our results hold only for $U$ sufficiently small, because they are based on perturbation-theory arguments that maximize the leading corrections of the ground-state energy as a function of $U$. Since these corrections of order $U^{2} \ln U$ will compete with order $U^{2}$ corrections for finite values of $U$, the phase separation discovered here may rapidly disappear as $U$ increases. Numerical evidence indicates that this is true for the densities between $\frac{1}{4}$ and $\frac{3}{4}$, but larger values of $U$ are necessary for the densities near 0 or 1 .

Furthermore, since the ground state is known to be either a different phase-separated state (nonneutral cases) or the most-homogeneous state (neutral case) for large $U$, the spinless Falicov-Kimball model must have a phase transition as a function of $U$. In the neutral case, when the ground state is not a phase-separated state, but is the Peierls-type state that maximizes the band gap at the Fermi level, it is possible that the ground state has no phase transitions for $0<U<+\infty$, since the small- $U$ ground state is identical to the large- $U$ ground state. We are unable to prove this conjecture here.

Our analysis was restricted to the spinless single-band Falicov-Kimball model, but the general ideas may also hold for more complicated models such as tertiary alloy problems (where $w_{i}$ would assume three different values) or the static Holstein model (where $w_{i}$ is continuous), but the determination of the maximal structure factor becomes much more complicated, since one must maximize with respect to both the phase and the amplitude, as opposed to maximizing only with respect to the phase, as we did here.

\section{ACKNOWLEDGMENTS}

We would like to acknowledge useful conversations with E. H. Lieb and D. Ueltschi. J.K.F. would like to acknowledge the hospitality of the Institut de Physique Théorique at the EPFL, where this work was started J.K.F. would also like to acknowledge the Donors of The Petroleum Research Fund, administered by the American Chemical Society, for partial support of this research (ACS-PRF No. 29623-GB6). 
${ }^{1}$ F. Bloch, Z. Phys. 52, 555 (1928).

${ }^{2}$ H. A. Kramers, Physica 2, 483 (1935).

${ }^{3}$ M. Morse, Phys. Rev. 35, 1310 (1930).

${ }^{4}$ R. de L. Kronig and W. G. Penney, Proc. R. Soc. London Ser. A 130, 499 (1930).

${ }^{5}$ J. C. Phillips and L. Kleinman, Phys. Rev. 116, 287 (1959); W. A. Harrison, ibid. 129, 2503 (1963).

${ }^{6}$ R. E. Peierls, Quantum Theory of Solids (Clarendon, Oxford, 1955), p. 108.

${ }^{7}$ H. Fröhlich, Proc. R. Soc. London Ser. A 223, 296 (1954).

${ }^{8}$ H. C. Longuet-Higgins and L. Salem, Proc. R. Soc. London Ser. A 251, 172 (1959).

${ }^{9}$ G. Benfatto, G. Gallavotti, and J. L. Lebowitz, Helv. Phys. Acta 68, 312 (1995).

${ }^{10}$ T. Kennedy and E. H. Lieb, Phys. Rev. Lett. 59, 1309 (1987); E. H. Lieb and B. Nachtergaele, Phys. Rev. B 51, 4777 (1995).
${ }^{11}$ J. L. Lebowitz and N. Macris, J. Stat. Phys. 76, 91 (1994).

${ }^{12}$ W. Hume-Rothery, The Metallic State (Oxford, Oxford, 1931).

${ }^{13}$ L. M. Falicov and J. C. Kimball, Phys. Rev. Lett. 22, 997 (1969).

${ }^{14}$ A. Sakurai and P. Schlottmann, Solid State Commun. 27, 991 (1978).

${ }^{15}$ T. Kennedy and E. H. Lieb, Physica A 138, 320 (1986).

${ }^{16}$ U. Brandt and R. Schmidt, Z. Phys. B 63, 45 (1986).

${ }^{17}$ J. K. Freericks and L. M. Falicov, Phys. Rev. B 41, 2163 (1990).

${ }^{18}$ Ch. Gruber, Helv. Phys. Acta 64, 668 (1991).

${ }^{19}$ U. Brandt, J. Low Temp. Phys. 84, 477 (1991).

${ }^{20}$ P. Lemberger, J. Phys. A 25, 715 (1992).

${ }^{21}$ Ch. Gruber, J. Lebowitz, and N. Macris, Europhys. Lett. 21, 389 (1993); Phys. Rev. B 48, 4312 (1993).

${ }^{22}$ Ch. Gruber, D. Ueltschi, and J. Jedrzejewski, J. Stat. Phys. 76, 125 (1994). 